\title{
The Challenges of Developing a Project-Based Self-Directed Learning Component for Undergraduate Medical Education
}

\author{
C. S. Keator ${ }^{1}$ - D. D. Vandre ${ }^{1}$ - A. M. Morris ${ }^{1}$
}

Published online: 2 August 2016

(C) International Association of Medical Science Educators 2016

\section{Introduction}

The successful implementation of a separate and dedicated self-directed learning (SDL) component within undergraduate medical education (UGME) curricula, especially those programs that do not use problem-based learning (PBL), has been an ongoing challenge for most institutions [1]. Project-based components have been implemented sparingly in the UK with some success, as these curricular approaches have been suggested to reduce student self-confidence but concomitantly provide students with a realistic vision for the challenges of lifelong learning [2]. At Western Michigan University Homer Stryker MD School of Medicine (WMed), we have implemented Explorations, a project-based SDL component of our UGME curriculum (please see Keator et al. [3]). This novel UGME approach is designed to help students think critically and use deductive reasoning, traits required for a lifetime of learning.

There are limited models to follow when designing novel approaches for SDL, and as such, there was no template to follow when we launched the project-based Explorations. To our knowledge, we are the first medical school in the USA to use a project-based SDL approach for UGME. Due to the lack of widespread use, implementing this project-based learning approach into the first 2 years of our UGME curriculum has resulted in both anticipated and unanticipated consequences. Thus, to help other institutions consider the feasibility of

C. S. Keator

christopher.keator@med.wmich.edu

1 Department of Biomedical Sciences, Western Michigan University Homer Stryker MD School of Medicine, Upjohn Campus, 1000 Oakland Drive, Kalamazoo, MI 49008, USA adopting this relatively novel method of SDL, the sections below highlight a few of the major administrative challenges we experienced during the first 2 years of the Explorations program at WMed.

\section{Explaining the Concept of Self-Directed Learning to New Medical Students}

One obstacle encountered by all new medical schools is conveying the concept and importance of SDL with students of the inaugural class. We initially developed Explorations to fulfill the Liaison Committee on Medical Education (LCME) educational directive (ED) 5-A, which prior to 2015 , listed "active learning" as a major requirement of that educational directive. Historically, ED-5-A had been one of the most cited standards for noncompliance, especially prevalent at those schools placed on "probationary status" during LCME accreditation curriculum reviews [4]. Active learning has been required to be part of the UGME curriculum for accreditation by the LCME for years, but unfortunately, a true definition for active learning never emerged from either the LCME or well-established medical education programs [5]. The LCME took notice of this problem, and more importantly, recently took proactive action to better define the requirements for SDL in UGME. In this regard, all active learning terminology was completely removed and omitted under redesign of accreditation standards and elements by the LCME (including LCME Element 6.3, formerly ED-5-A) and instead was replaced with the well-defined concept of "self-directed learning" (www.lcme.org). The revamped LCME guidelines now encourage all programs to clearly outline and explain the importance and goals of all SDL activities, and integrating SDL throughout the 4 years of UGME is recognized as a top priority by all medical schools, especially new schools building a curriculum from scratch. 
Our inaugural class initially struggled with understanding the overall purpose of Explorations. In retrospect, this challenge arose because we did not adequately orient them to the importance of SDL as a required competency, i.e., why they were being challenged to think and independently identify learning topics of their own choice. This problem was most likely compounded by the lack of guidance provided by upperclassmen, since similar concerns about SDL have been observed by those programs utilizing electronic distance learning [6]. Shortly, after the LCME released the new standards and terminology (approximately at the beginning of year 2 of the WMed UGME), we made a concerted effort to better inform the inaugural class of the required competency in SDL and the role that Explorations plays in meeting this competency. This resulted in better acceptance of Explorations by the students. We subsequently developed a unique series of events, which we refer to as "Orientation to SDL" (Phase 1 of Explorations), for our second cohort of medical students. As a result, when comparing our inaugural class with our second class, the second class has a substantially better understanding of the purpose of Explorations, which has contributed to improved SDL skills as evidenced by the creation of higher quality learning products (i.e., deliverables) much earlier in the curriculum than our inaugural class.

We are also fortunate, in that now we can show incoming students various examples of SDL projects completed by our upperclassmen. These examples have been invaluable for illustrating the freedom granted in our project-based SDL component. Moving forward, we are also in the process of creating a digital platform to archive and share these projects on our WMed website. This will permit all members of our WMed family, including community faculty and non-academic staff, to view the Explorations projects. Importantly, our students will be able to retrieve and review any of these projects during all 4 years of UGME. In essence, we are creating a digital portfolio that individual students can use to gauge their progress and reflect on their journey of lifelong learning. Using eportfolios in learning, including UGME, have been shown to be beneficial for students of this current Millennial generation [7-10]. The concept of keeping a portfolio of SDL-driven projects is a unique difference that sets this project-based component apart from PBL, and we feel that this capability further promotes the use of this type of SDL approach for students of the modern digital age.

We recommend that if you embark on implementing this project-based type of SDL curricular component that you consider the following: (1) fully describe the purpose of SDL, including the impact of using these skills for lifelong learning and the requirement that your students meet this competency; (2) clearly outline a well-defined set of expectations for student involvement; and (3) provide students with examples of the types of deliverables they could create, recognizing that even with examples, patience is needed as students work through the process of creating projects. Providing examples will further delineate expectations for how today's students can use modern technology to extrapolate science to medicine.

\section{Objectively Tracking Student Progress and Assessment}

The manner in which WMed tracks student performance and progress for the Explorations component continues to evolve, largely because the amount of creative freedom afforded by students is enormous. This freedom results in an open palette for learning topics, which translates into an equally large range of the types of deliverables that can be (and are routinely) created. Thus, student progress during development of an Explorations project needs to be appropriately monitored to keep students on track.

Students receive constructive feedback from individual faculty facilitators and from the Director of Explorations. Students themselves also openly critique their team performance during presentations and evaluate one another through confidential peer reviews distributed twice yearly by our Office of Student Affairs. Course assessment is also conducted by our students after each Explorations learning event, and this information is part of the biannual WMed Curriculum Committee review process. Thus, the continuous quality improvement mechanism at WMed ensures that any student issues with Explorations are quickly identified.

We use our in-house Explorations facilitator assessment form to track the types of feedback being given by our facilitators across all groups. We have noticed that the type of feedback (e.g., comments regarding the complexity of the information resources used for projects) has remained relatively consistent as the students' SDL skills have evolved. This simple tracking mechanism is important, because it gives the Director of Explorations the ability to simultaneously examine and track the progress by all groups, and thereby intervenes if any group is consistently underperforming in acquiring or using the skills needed to meet this competency. Objective assessment and student feedback fuel change, and therefore we have accepted that this is an evolving process that will continually help Explorations improve.

For anyone considering this type of project-based SDL, we recommend developing an in-house assessment rubric, specific to the expectations of the course that can be used to easily identify any problem areas as they develop. Open lines of communication must be maintained between the course director, the faculty facilitators, and the students involved in the course, to rapidly identify problems and make changes when they arise. While students must be held accountable for a lack of effort, this must be balanced by providing them with additional opportunities to improve their SDL skills. In this regard, it is critical to remember that these students are grappling with comprehending and transforming highly complex scientific information into layman's terms, which can be a challenge 
for even the most seasoned scientists and educators. Finally, you need to establish a graduated set of expectations, because based on our experience over the course of the first 2 years of UGME at WMed, each student's SDL skills (and group efforts) improved over time and continue to evolve. It is necessary to establish and define reasonable expectations that are communicated to the students prior to implementation of the course for a consistent process across the entire UGME experience, and it is imperative that the facilitators maintain uniformity and objectivity in evaluating the process.

\section{Maintaining a Consistent Experience for All Students}

Small groups naturally encourage constructive teamwork, but the ability to develop and maintain consistency is a logistical administrative challenge inherent to all small group teaching [11], and Explorations is not immune to these problems. There are a number of inherent independent variables (those that cannot be manipulated) that are part of Explorations groups, including the following: (1) student premed experience, (2) student ability to interpret complex scientific information, (3) facilitator expertise, (4) facilitator personal preferences, and (5) group dynamics. In addition, because of the independent nature by which groups identify topics for the Explorations projects and the differences in types of projects created, there is also a significant difference between the activities that individual students experience between groups. Given all of the variables inherent to small group activities, achieving and maintaining a consistent experience across all Explorations groups requires constant monitoring and is therefore an ongoing process. Collectively, we have focused on ensuring that each group follows a consistent process to achieve their varied project outcomes.

We initially planned to have students switch Explorations groups four times during their first 2 years of UGME. This strategy paralleled the number of times our students switch team-based learning groups, and therefore seemed logical. However, after the first 6 months of Explorations, we decided that students should remain in the SDL groups for an entire year, thereby allowing each student the chance to develop their individual SDL skills in the comfort of the same group for a longer period of time. Thus, in our UGME curriculum, the students are reassigned to new groups only once, after the groups complete their first presentation to a large group (i.e., the other student groups) at the end of year 1. Keeping groups together for a longer period of time allows each group to recognize each member's strengths and weaknesses, thereby enhancing the consistency across groups and improving the value of the learning process for all students.

In setting up the Explorations groups, we currently distribute our non-science majors equivalently across all groups but have found that this alone is not sufficient to balance the group dynamics for optimum performance in a project-based SDL component. Moving forward, we plan to better account for the student's premed experience by evaluating student attributes prior to assigning students to the groups. We are in the process of developing a screening tool to evaluate each student's premedical exposure to (a) peer-reviewed literature, (b) basic research, (c) clinical experiences, and (d) life experiences (e.g., foreign travel). We believe that accounting for these additional attributes will help us better ensure that individual student capabilities within our groups are more equally distributed, an aspect that we overlooked when developing the Explorations groups for our inaugural class. Collectively, we are striving to have all of the Explorations groups in future classes progress with less variability, thereby helping our students more consistently acquire and develop the skills required for SDL.

\section{Organizing, Administrating and Improving a Project-Based SDL Component}

The three challenges listed above (student buy-in, objectively tracking student progress, and achieving consistency among student groups) must be addressed for all small group components $[12,13]$ and therefore are not unique to Explorations. However, in a project-based SDL component like Explorations, those three challenges are amplified due to the large degrees of freedom granted to both facilitators and students. The independence of the direction and topics that groups pursue requires a dedicated course director to continually monitor progress and encourage a cohesive effort from all faculty involved in developing, running, and refining a project-based SDL component.

The amount of time to direct and manage this project-based component is substantial; this increased time commitment is the result of granting autonomy to the student's time and freedom necessary for them to take ownership of their SDL. This independence has resulted in the creation of wonderfully diverse projects, but this large diversity results in the need for a common review of all projects (by the course director), a higher level of scrutiny needed to clearly determine how well each group is progressing within the competency of SDL. The course director is also charged with maintaining consistency among facilitators, which is also an ongoing administrative challenge due to the freedom afforded by the facilitators in guiding their groups.

The faculty facilitator may unintentionally influence group dynamics in any type of small group learning [13, 14], and facilitator influence was a pressing concern when we first started Explorations. Faculty in all institutions come from different backgrounds with varying levels of training, and therefore it cannot be assumed that every facilitator will set equal expectations for their groups. We are fortunate (at WMed) in that our facilitators are all currently based in one department, which allows facilitators ample opportunities to assess and 
discuss their facilitation approaches and evaluate student group performance on a regular basis. Explorations facilitators also often substitute for one another and therefore facilitate different groups, providing an opportunity for students to experience a different facilitator's viewpoint and also providing our faculty facilitators better insights into how multiple groups are handling the processes. The director also routinely reinforces both the purpose and goals established for Explorations, which provides additional opportunities for faculty to develop their facilitation skills. In this regard, we have noticed that as each faculty member becomes more accustomed to facilitating Explorations sessions that student concerns regarding consistency among groups has almost disappeared.

Importantly, a project-based SDL component flourishes with student autonomy but still requires consistency. Autonomy and consistency often exert opposing forces, and therefore a proper balance of expectations must be formed between the facilitator and students. The director and all faculty facilitators must maintain objectivity and evaluate both student performance and progress on the quality improvements in their SDL process. In a project-based component, quality improvement and level of learning during a project may not be directly reflected in all of the finished deliverables. For example, it is near impossible to adequately gauge the "quality" or "value" of a pamphlet aimed at informing elderly patients about hip replacement when that deliverable is compared with an animated video developed to teach medical students about the microbiota of the gut. Further, the director must enforce the requirement that facilitators evaluate and assess the entire process of SDL and not just the finished product. This protects the SDL competency from subjective personal preferences of the facilitators, which can unintentionally cloud judgment of the content. In a project-based approach, this subjectivity can arise from the choice of topic or mode of distribution. For instance, facilitator A prefers posters, facilitator B prefers videos, and facilitator C prefers slideshows (etc.). Inherently, facilitator evaluation cannot reflect those personal preferences. Collectively, all these differences require a novel management strategy, one tailored specifically for a project-based modality like Explorations.

\section{Summary}

Many aspects of medical curricula improve upon themselves, especially those components geared toward SDL [15]. Collectively, a modern (project-based) SDL approach requires constant administrative assessment, evaluation, and refinement to ensure students are progressing at an acceptable rate in acquiring the skills required for a lifetime of learning. As we obtain student feedback and learn more about the effectiveness of Explorations, we expect a continual cycle of improvement each year. Ultimately, (at WMed) we are aiming to consistently improve our students' appreciation of Explorations, providing them with the opportunities to help themselves develop strong SDL skills and in the process preparing them to be lifelong adult learners. Thus, the SDL goals that we have set for students at WMed, which are also relevant for all types of SDL and promote lifelong learning, are listed below:

1) Identify gaps in knowledge as related to science and medicine

2) Identify a learning topic to address gaps in knowledge

3) Develop learning objectives for identified learning topic

4) Retrieve relevant information resources

5) Evaluate information sources

6) Use evidence-based medicine to distinguish facts from fiction

7) Collaborate with peers to create a deliverable

8) Provide constructive feedback to peers

9) Receive critical feedback from a diverse audience

10) Practice reflection to independently identify learning preferences, strengths, weaknesses, and personal bias

In sum, the skills and perseverance to practice the art of SDL for a lifetime of learning are actually quite basic, because from a Gestalt view, SDL is merely turning curiosity of the unknown into the search for and understanding of "new" information. The digital age has presented medical education with the modern conveniences of unlimited access to online resources, the ease to create various types of multiple types of products (slideshows, pamphlets, etc.), the capability of setting up an electronic portfolio, and more. Thus, modern SDL for the Millennial students of today needs to encompass those conveniences during UGME, because the concepts behind those conveniences will eventually evolve into clinical expectations when those students of today start practicing medicine tomorrow.

\section{References}

1. Murad MH, Coto-Yglesias F, Varkey P, Prokop LJ, Murad AL. The effectiveness of self-directed learning in health professions education: a systematic review. Med Educ. 2010;44:1057-68.

2. Whittle SR, Murdoch-Eaton DG. Student-selected projects: can they enhance lifelong learning skills? Med Teach. 2002;24:41-4.

3. Keator CS, Dickinson BL, Lackey WL, Morris A, Quesnelle KM, Riddle DR, Sheakley ML, Vanden Heuvel GB, Vandre DD. Explorations: a new approach to self-directed learning in a competency-based curriculum. Med Sci Educ. 2016; doi:10.1007 /s40670-016-0293-0.

4. Hunt D, Migdal M, Waechter DM, Barzansky B, Sabalis RF. The variables that lead to severe action decisions by the liaison committee on medical education. Acad Med. 2016;91:87-93. 
5. Kaufman C, Conway S, Gallagher KE. Health information resources: tradition and innovation in a medical school curriculum. Med Ref Serv Q. 1999;18:11-23.

6. Delgaty L. Twelve tips for academic role and institutional change in distance learning. Med Teach. 2015;37:41-6.

7. Barnes SP, Torrens A, George V, Brown KM. The use of portfolios in coordinated school health programs: benefits and challenges to implementation. J Sch Health. 2007;77:171-9.

8. Bok HG, Teunissen PW, Favier RP, Rietbroek NJ, Theyse LF, Brommer H, Haarhuis JC, van Beukelen P, van der Vleuten CP, Jaarsma DA. Programmatic assessment of competency-based workplace learning: when theory meets practice. BMC Med Educ. 2013;13:123.

9. Hall P, Byszewski A, Sutherland S, Stodel EJ. Developing a sustainable electronic portfolio (ePortfolio) program that fosters reflective practice and incorporates CanMEDS competencies into the undergraduate medical curriculum. Acad Med. 2012;87:744-51.

10. Williams BC, Mullan PB, Haig AJ, Malani PN, Perry JS, Riba M, Williams JM, Kolars JC, Mangrulkar RS. Developing a professional pathway in health equity to facilitate curricular transformation at the University of Michigan Medical School. Acad Med. 2014;89:1153-6.

11. Morrow G, Rothwell C, Wright P. Self-directed learning groups: a vital model for education, support and appraisal amongst sessional GPs. Educ Prim Care. 2012;23:270-6.

12. Fleiszer DM, Posel NH. Development of an undergraduate medical curriculum: the McGill experience. Acad Med. 2003;78:265-9.

13. Tufts MA, Higgins-Opitz SB. What makes the learning of physiology in a PBL medical curriculum challenging? Student perceptions. Adv Physiol Educ. 2009;33:187-95.
14. Shokar GS, Shokar NK, Romero CM, Bulik RJ. Self-directed learning: looking at outcomes with medical students. Fam Med. 2002;34:197-200.

15. Colbert CY, Graham L, West C, White BA, Arroliga AC, Myers JD, Ogden PE, Archer J, Mohammad ZT, Clark J. Teaching metacognitive skills: helping your physician trainees in the quest to 'know what they don't know'. Am J Med. 2015;128:318-24.

\section{Contributors}

Dr. Keator was appointed as the Director of Explorations during the rollout period of Explorations for the WMed Inaugural Class of 2018 and has developed many of the improvements in place for the Class of 2019. Dr. Vandre is the Chair of the Department of Biomedical Sciences, was recently appointed as the Associate Dean of Research, and is involved heavily in developing the curriculum for the first 2 years of UGME. Dr. Morris is the Assistant Dean of the Foundations of Medicine, developed the original vision of the project-based Explorations, and is charged with developing and integrating the various components of UGME into a unified 4-year experience for all students. All three of these individuals have contributed to the development, implementation, administration, and ongoing evolution of Explorations. All three also serve on the WMed Evidence-Based Medicine (EBM) Working Group, charged with integrating EBM throughout the 4 years of UGME, which has further strengthened the role of SDL (via EBM and Explorations) within the WMed curriculum. 